Japanese Psychological Research

1081 , Vol. 23, No. 2, 88-100

\title{
Availability of objective information and social comparison behavior ${ }^{1}$
}

\author{
TOSHITAKE TAKATA ${ }^{2}$ \\ Department of Psychology, Gunma University, Maebashi 371 \\ HARUO HAYASHI \\ Waseda University ${ }^{3}$
}

Three experiments tested basic hypotheses of the social comparison theory regarding the relationship between the availability of objective means for self-evaluation and the occurrence of social comparison. Undergraduate subjects were asked to answer 20 items of a musical aptitude test. When the subjects pushed the "social comparison button," they could see other subjects' answers displayed on an electric panel in their cubicles. When the information about the correct answer was unavailable, the subjects pushed the button most frequently to compare their answers with others', especially when the criterion of correct answers was social consensus. Social comparison of this type was regarded as informational social comparison. However, the subjects showed a considerable amount of social comparison even when they received the objective information, if the subjects' self-esteem was enhanced either through the objective information or through high self-esteem manipulation prior of the testing. Social comparison of this type was regarded as normative social comparison.

Key words: experimental social psychology, social behavior, social comparison processes, ability comparison, information seeking, self-evaluation, self-enhancement.

There is no doubt that Festinger's (1954) theory of social comparison processes is one of the seminal theories in social psychology. Strangely enough, however, there have been few empirical studies about the theory, especially about its basic assumptions. In a recent book by Suls and Miller (1977), a sizable sum of experimental data had been accumulated, and there was an attempt to reformulate Festinger's original theory from the standpoint of attribution theory in that book (Goethals \& Darley, 1977). But most of these experiments

1 A part of data reported here was presented at the 23rd and 24th annual conventions of the Japanese Group Dynamics Association in 1975 and 1976.

2 The help of Mr. Yoshiyuki Matsumoto, Professor Hitoshi Hashimoto, and Professor Masatoshi Tomita in making this study possible is gratefully acknowledged. We would like to thank Dr. Barry Collins, Dr. Harold Gerard, and Dr. Irwin Mahler for commenting on earlier versions of this manuscript.

${ }^{3}$ Now at University of California, Los Angeles. have been concerned with the similarity hypothesis, that is, "the tendency to compare oneself with some other specific person decreases as the difference between his opinion or ability and one's own increases (Festinger, 1954, Hypothesis III)." They take for granted the arousal of a social comparison need. Experimental investigations have not yet tested Festinger's first two hypotheses, that is, "there exists, in human organism, a drive to evaluate his opinions and abilities (Hypothesis I)" and "to the extent that objective, non-social means are not available, people evaluate their opinions and abilities by comparison respectively with the opinions and abilities of others (Hypothesis II)."

The findings that people affiliate with others in order to reduce uncertainty about their emotional states (Gerard \& Rabbie, 1961), their opinions (Radloff, 1961), their abilities (Singer \& Shockley, 1965), and their personality traits (Takata, 1971) are generally interpreted as the indirect con- 
firmations of the Festinger's first two hypotheses. But these findings remain somewhat equivocal because of such confounding factors-other than self-evaluative social comparison need itself-as fear reduction and situational factors involved in face-to-face interaction.

Among a few exception, Takata (1974) conducted a direct experimental test of Festinger's first two hypotheses. In his experiment, which was presented in the guise of a test of perceptual abilities, undergraduate subjects were seated in individual cubicles and asked to make auditory judgement. When subjects pushed the "social comparison button", they could know the "other participants' answers" displayed on an electric panel in the subjects' cubicles. Some of the subjects were informed on each trial whether their answers were correct (information conditions), and some were not informed (no information condition). In the no information condition where objective criteria for the correctness of one's answers were unavailable, subjects pushed the social comparison button more than did the subjects in information conditions.

Furthermore, information conditions were divided into an information-correct and an information-incorrect condition. The subjects in information-correct condition, regardless of their actual answers, received the false feedback that their answers were correct in two-thirds of the total questions. The subjects in this condition pushed the social comparison button as much as the no information subjects. By contrast, in the information-incorrect condition, where subjects learned that two-thirds of their answers were incorrect, the subjects pushed the button on few trials.

These results appear to confirm Festinger's basic hypotheses in some respects, but to contradict them in other respects. Festinger's first two hypotheses were supported by the social comparison responses of the no information subjects which indicate their needs to evaluate their abilities by comparing their own judgements with others'. In contrast, the finding that the subjects in information-correct condition tried to learn the others' answers disconfirmed the notion that social comparison is used for accurate self-evaluation. If social comparison does serve only for selfevaluation, there should be no need for social comparison responses in informationcorrect condition where the immediate self-evaluative needs were directly satisfied by the objective information. Thus, the considerable amount of social comparison responses in this condition suggest an additional function of social comparison other than self-evaluation. Furthermore, the results of information-incorrect condition might be interpreted as, not only due to mere satiation of self-evaluative need, but also due to avoidance of social comparison.

Such reasoning led Takata (1974) to hypothesize that there exist two functions in social comparison processes: namely, the informational function and the normative function. This dichotomy is consistent with Latané's (1966) distinction of comparison motives, that is, self-evaluation versus self-enhancement. The informational comparison is defined as the comparison that works as an informational basis for accurate self-evaluation under high uncertainty. While the normative comparison is defined as the one which enhances one's self-concept in order to conform with the positive expectations of oneself and / or another (Deutsch \& Gerard, 1955). We assume that the normative comparison consists of two kinds of behavioral manifestation, that is, the search for further information and the avoidance of it. The search for further information makes the active self-enhancement possible as is the case in the information-correct condition in which subjects were able to enhance their self-concept by affirming their own good performances in comparison with other participants. As with the information-incorrect condition, the avoidance of social comparison might have helped the 
subjects to maintain their self-concept through passive self-defensiveness (for thorough discussion, see Takata \& Takata, 1976).

Unfortunately, the experiment by Takata has some methodological shortcomings and thus his findings remain somewhat ambiguous. For example, the "other participants' answers" were prearranged to change unanimously throughout the experiment. Some of the experimental tasks were so easy that information-incorrect subjects soon became suspicious of the experimental manipulations. Moreover, the research design for information conditions did not permit a clear distinction between two functions of social comparison responses. The objective information was useful not only for informational comparison but also for normative comparison because it was of a self-enhancing nature in information-correct condition and of a selfdevaluating nature in information-incorrect condition.

These problems led to the development of the current three experiments which overcome the abovementioned methodological shortcomings. In Experiment I, the effect of availability of the objective information upon the informational social comparison behavior was tested while keeping the normative implications of objective information at a minimum. Experiment II was designed to replicate the Takata's (1974) experiment that the normative social comparison behavior did occur even when self-evaluation need was satisfied by the objective information. Experiment III examined the effect of subjects' selfesteem as one of the determinants of normative social comparison behavior.

\section{Experiment I}

Festinger's first two social comparison hypotheses were tested directly in this experiment; normative social comparison was kept at a minimum level. It was predicted that people would compare their own at- tributes with others' for the purpose of selfevaluation under high uncertainty due to the lack of objective means for self-evaluation. High uncertainty, in this experiment, was operationally defined in terms of the availability of feedback on correct answers and the nature of task the subjects went through.

We set up two kinds of experimental task with equal task difficulty; physical measurement tasks and social consensus tasks. The physical measurement task are the ones in which the criteria for correct answers can be determined ultimately by means of physical measurement such as an oscillograph and a chronograph. In these task, comparing one's own answers with others' might be useful as only a convenient and costless check on what right answers are. The social consensus tasks are the ones in which the criteria for correct answers are primarily based on the consensus of peoples. In these tasks, social comparison might be of most importance.

In this experiment, we hypothesized that there would be greater social comparison responses when objective information was unavailable than when it was available (Hypothesis 1). We also hypothesized that social comparison responses would be prominent when the correct answer for the task was determined by the social consensus than when it was determined by the physical measurements (Hypothesis 2).

\section{Method}

Subjects. The subjects were 46 male and female undergraduates taking an introductory psychology class at Waseda University. The data from one male subject were discarded because of his suspicion of the experimental manipulations. The postquestionnaire indicated that there were no systematic differences among the subjects' musical backgrounds.

Design and overview. The availability of the objective information and the criteria for correctness of answers were manipulated in $2 \times 2$ factorial design. The subjects, 
in groups of $\operatorname{six}^{4}$, were administered a series of musical aptitude test items under the guise of a validation study of a newly developed test. The availability of the objective information was manipulated by either (a) informing subjects immediately after they answered or (b) not informing them whether their answers were correct. The criteria for correctness was manipulated by either administering the test items (a) for which correctness could be measured physically or (b) for which correctness was based on the social consensus about what is good music. The subjects could see signal lights on an electric panel that appeared to represent five other participants' answers if the subjects pushed a key named the " information request key" in their cubicle. The informational social comparison need was measured chiefly by the number of the subjects' depressing this key.

Procedure. Subjects were seated in individual cubicles where they could see an experimenter but not each other. In each cubicle, there was an apparatus which consisted of an electric panel of 12 signal lights arranged in two rows of six, response keys, an information request key, feedback lamps, and a headphone. After the introductory remarks regarding the purpose of the experiment, the experimenter began to explain the alleged functions of the apparatus as follows:

The test will be administered through this apparatus named " Group Response Analyser" which was originally designed for a different purpose. This is because the final test form has not yet completed. Let me explain the functions of this apparatus step by step. When you answer the question by a response key, your answer is displayed by a signal light on your panel and on the experimenter's master panel. The other participants' answers will be displayed

- Unless six subjects showed up, confederates were used to fill up. on your panel if you push the information request key. Furthermore, the feedback lamps tell you whether your answers are right or wrong, if correct answers are programed in advance. A green lamp means your answer is correct, while a red one means incorrect.

A handout entitled "how to use Group Response Analyser" and some practice trials made subjects familiar with the apparatus.

What appeared to be the others' answers and the objective information (feedback lamp) were manipulated independetnly in a predetermined manner by the another experimenter behind the scene. Five others' answers were randomized trial by trial so that we could reduce the unanimous answers. In the information (Inf) conditions, where the objective information was available, the subjects received the feedback that their answers were correct on 10 out of 20 trials regardless of their actual responses. Thus, the information gave the subjects the impression that their performances were neither very good nor very poor, in order to keep normative social comparison at a minimum. In the no information (No Inf) conditions where such information was unavailable, the experimenter told the subjects that the feedback lamps were not used in the experiment.

In the conditions where the criteria for correctness of their answers was based on physical measurement (physical measurement (Physic) condition), we administered 20 test items from the Seashore Measures of Musical Talents (Seashore, Lewis , \& $^{2}$ Seatveit, 1960). The first items were selected from the "rhythm" test, and the last 10 items from the "time" test. In the former, subjects were to indicate whether the two rhythmic patterns of seven notes in $4 / 4$ time in pair were the same or different. In the latter, subjects were to determine whether the second tone was longer or shorter than the first of a pair of different durations. In the conditions 
where the criteria for correctness was based on social consensus (social consensus (Soc) condition), we administered 20 test items from the Wing Standardised Tests of Musical Intelligence (Wing, 1960). The first and second 10 items were selected from the "rhythm appreciation" test and the "phrasing appreciation" test respectively. In both tests, subjects were to choose between a balanced and an unbalanced rhythm or phrasing of a pair of melodies played on the piano.

Subjects were given through the headphone a series of 20 items as 20 second intervals. During this interval, they were asked to indicate their answers by the response keys and they were allowed to push the information request key if they wanted to see the others' responses. Furthermore, after each trial, subjects were to rate their confidence on their answers on a 7-point scale. After the test, subjects were asked to fill out a questionnaire including an item asking the extent to which they generally desired to see the others' answers. Then they were debliefed, sworn to secrecy, and dismissed.

\section{Results and Discussion}

Manipulation checks. In order to assess how the objective information was interpreted by the subjects, an item of the postexperimental questionnaire asked them to estimate the percentage of their correct answers. In the Inf conditions, the mean ratings were about $50 \% \quad(51.0 \%$ and $51.7 \%$ for Physic and Soc conditions respectively). The mean ratings in the No Inf conditions were also about $50 \%(57.1 \%$ and $56.0 \%$ for Physic and Soc conditions respectively). There were no statistically significant differences among these ratings. Thus, we could assume that the objective information in this experiment was neither self-enhancing nor self-devaluating.

Subjects were also asked in another item of the post-questionnaire to evaluate to what extent the test items has an objective correctness criterion. The mean rating of
Table 1

Mean amount of social comparison in Experiment $I$

\begin{tabular}{lccc}
\hline \multirow{2}{*}{ Groups } & $\mathcal{N}$ & \multicolumn{2}{c}{ Measure } \\
\cline { 3 - 4 } & & Behavioralt & Cognitivett \\
\hline $\begin{array}{l}\text { Physic } \\
\text { No Inf }\end{array}$ & 14 & 10.43 & 4.50 \\
Inf & 10 & 7.00 & 3.90 \\
\hline Soc & & \\
No Inf & 10 & 15.70 & 5.50 \\
Inf & 12 & 6.50 & 4.42 \\
+ A score of 20 is maximum frequency. \\
t† A score of 7 is maximum desire to see others' \\
answers.
\end{tabular}

the Physic conditions tended to be higher than that of the Soc conditions (4.75 and 4.05 in a 7 -point scale for the former and the latter condition, respectively: $F(1,42)$ $=3.18, p<.08$ ). The subjects in the Soc conditions appeared to be less dependent on objective criterion for correctness. Consequently the experimental manipulations were successful.

Social comparison responses. Table 1 shows the mean number of the trials in which subjects pushed the information request key (behavioral index) and the mean ratings of subjects' desire to see others' answers (cognitive index). A $2 \times 2$ analysis of variance revealed that the main effect of information availability was significant for both behavioral index $(F(1,42)=10.85$, $p<.005)$ and cognitive index $(F(1,42)=$ $3.96, p<.06)$. As predicted in Hypothesis 1 , these results showed that subjects in the No Inf conditions pushed the social comparison key and desired to see the others' answers more than those in the Inf conditions. Thus, these results supported $\mathrm{Hy}$ pothesis 1 .

Although the information availability $x$ criteria for correctness interaction did not reach statistical significance, the simple effect of criteria for correctness at the No Inf conditions indicated that the social comparison key was pushed significantly 
more in the Soc condition than in the Physic condition $(F(1,42)=3.96, p<.06)$. As for the cognitive measure, we also had the similar significant simple effect at the No Inf conditions $(F(1,42)=5.72, p<.05)$. Furthermore, the post-questionnaire item about the perceived task difficulty showed no significant differences between the two criteria for correctness conditions. Thus, these differential effects of the criteria for correctness cannot be attributed to the difference of task difficulty. In the light of these results, we think that Hypothesis 2 was partially confirmed.

Changes over trials. Do social comparison responses increase or decrease in process of trials? Actually Takata (1974) found some changes over trials. In order to examine this posibility, the mean frequencies of pushing the information request key were devided into four blocks of five trials each. A $2 \times 2 \times 4$ analysis of variance revealed significant main effect of trial block $(F(3,126)=17.71, p<.005)$ and the information availability $\times$ trial block interaction $(F(3,126)=5.55, p<.005)$. Subjects generally showed an increase in social comparison responses. However, the increase in social comparison responses was significant for only the subjects in the Inf conditions $(F(3,126)=9.43$ for the Soc condition; $F(3,126)=12.66$ for the Physic condition, respectively. Both $p s<.005$ ).

\section{Experiment II}

As indicated earlier, social comparison behavior would occur if subjects' self-evaluative need was satisfied with the objective information which enhanced their selfesteem. The purpose of this experiment is to replicate Takata's (1974) findings with the present research paradigm. Thus, the effect of self-enhancing information upon social comparison behavior was examined in contrast to the effect of self-devaluating information. Specifically the following hypotheses were tested: On the one hand, if the available objective information sup- ported many of subjects' answers, it thereby enhanced their self-esteem; so many social comparison responses, as many as those of the subjects having no information, would occur in spite of the feedback that satisfied the immediate self-evaluation need (Hypothesis 3). On the other hand, if many of subjects' answers were not supported by the objective information and the subjects' self-esteem was threatened, social comparison responses would be suppressed (Hypothesis 4).

\section{Method}

Subjects. The subjects were 39 male and female undergraduates from the same population as in Experiment I. The data from one male subject were discarded because of his suspicion about the apparatus.

Procedure. Three experimental conditions - no information (No Inf), self-enhancing information (Enhanc Inf), and self-devaluating information (Devaluat Inf) conditions-were used. The experimental procedure was identical with that of the social consensus conditions in Experiment I except for the following changes ${ }^{5}$. Since the objective information was supposed to enhance or threaten subjects' self-concept, "feedback lamps" indicated that the subjects' answers were right on $70 \%$ out of 20

5 In Experiment I, the effects of the availability of the objective information upon social comparison responses were more prominent in the Soc condition than in the Physic condition. Thus only the former condition was used in the subsequent experiments. In Experiment II, before the musical test, subjects were asked to answer another musical test in melodic taste which was adopted from the Kwalwasser-Dykema Music Test (Kwalwasser \& Dykema, 1930) under the guise of establishing the criterion for the newly developing test which were to follow. The subjects' performances of this test were not fedback. The addition of this procedure enabled this experiment to be used as control conditions for Experiment III described later. Neither the subjects' actual performances of the KwalwasserDykema Test nor their evaluations of their performances showed any systematic differences among the experimental conditions. 
trials in the Enhanc Inf condition, and only $30 \%$ in the Devaluat Inf condition.

\section{Results and Discussion}

Manipulation checks. If the manipulation of the objective information was effective, subjects should estimate the percentage of their correct answers as follows: $50 \%$ for the No Inf, $70 \%$ for the Enhanc Inf, and $30 \%$ for the Devaluat Inf condition ${ }^{6}$. The means of these estimates obtained in the post-questionnaire were $57.8 \%, 58.0 \%$, and $26.4 \%$, respectively. Although the mean estimate of Enhanc Inf condition was considerably lower than expected, there were significant differences among these three conditions $(F(2,36)=26.48, p<.005)$.

Another item in the post-questionnaire replicated the same trend. Subjects were also asked to evaluate their test performances as compared with their perception of their own musical ability. The mean ratings of each condition in a 7 -point scale turned out to be 3.22 for the No Inf, 4.10 for the Enhanc Inf, and 2.18 for the Devaluat Inf. In spite of the significant main effect $(F(2,36)=10.44, p<.005)$, the mean rating of the Enhanc Inf condition barely exceeded the midpoint indicating that the subjects did not regard their performances as very good. In sum, the objective information manipulation tended to be effective in general. Its effectiveness for the Enhanc Inf condition, however, remained somewhat doubtful in contrast with both the No Inf and the Devaluat Inf condition.

Social comparison responses. The mean frequencies of pushing the social comparison key are shown in Table 2. An analysis of variance reveals a significant main effect $(F(2,36)=10.32, p<.005)$. In the No Inf condition, the subjects pushed the information request key significantly more than those in other two conditions (both $p s<.05$, by Newman-Keuls test), as were

- In the No Inf condition, an estimate of about $50 \%$ was expected according to the results of the No Inf conditions in Experiment I.
Table 2

Mean amount of social comparison in Experiment II

\begin{tabular}{lccc}
\hline \multirow{2}{*}{ Groups } & \multirow{N}{*}{$\mathcal{c}$} & \multicolumn{2}{c}{ Measure } \\
\cline { 4 - 4 } & & Behavioral & Cognitive \\
\hline No Inf & 18 & 19.21 & 5.61 \\
Enhanc Inf & 10 & 10.10 & 4.40 \\
& $(6)$ & $(14.83)$ & $(5.83)$ \\
Devaluat Inf & 11 & 10.45 & 4.09 \\
& $(9)$ & $(8.78)$ & $(3.67)$
\end{tabular}

Note. Numbers in parentheses indicate results of internal analysis.

the comparable conditions in Experiment I. The cognitive measure also showed similar trends $(F(2,36)=2.78, p<.08)$, in that only the subjects in the No Inf condition desired to see others' answers more strongly than those in other two conditions.

These results did not support our $\mathrm{Hy}$ pothesis 3 , which predicted so many social comparison responses in the Enhanc Inf condition as those of the No Inf condition. As mentioned earlier, however, this may be partly due to those subjects in the Enhanc Inf condition who failed to perceive the objective information accurately and who thereby evaluated their performances being poor. In order to check this possibility, an internal analysis was done in which we discarded the data of the six subjects in the information conditions whose estimates of their performances were extremely low in the Enhanc Inf or high in the Devaluat Inf condition. Once these subjects were eliminated, the mean estimate of the Enhanc Inf condition turned out to be $68.8 \%$, which was close to the expected figure. Numbers in parentheses in Table 2 showed the social comparison responses of each condition in the internal analysis.

The internal analysis showed that three conditions differed significantly in the hypothesized direction in terms of social comparison responses $(F(2,30)=12.54, p<$ .005 for the behavioral measure; $F(2,30)=$ 
$5.28, p<.05$ for the cognitive measure). The subjects who perceived accurately the information conveying their superior test performances showed significantly more social comparison responses than those who received the self-devaluating information did ( $p s<.05$ for both measures by Newman-Keuls tests). The social comparison responses in the Enhanc Inf condition were also significantly higher than those in the comparable Inf condition of Experiment I ( $p \mathrm{~s}<.05$ for both measures by $t$ tests). Thus, this internal analysis tends to support Hypothesis 3.

Comparing the social comparison responses in the Devaluat Inf condition in the internal analysis with those in the comparable Inf condition of Experiment I, there were no significant differences between two conditions. As far as the social comparison responses were concerned, these results gave no support for our $\mathrm{Hy}-$ pothesis 4 which predicted the supression of social comparison responses when the subjects received the self-devaluating information. However, in an item of the post-questionnaire, the subjects in the Devaluat Inf condition rated that the criterion for determining correct answers in the test was less reliable than did those in other two conditions. (The mean ratings in a 7point scale were $4.05,5.17$, and 3.54 for the No Inf, the Enhanc Inf, and the Devaluat Inf condition, respectively: $F(2,36)$ $=2.80, p<.08$.) This may be interpreted as an indication of subjects' self-defensive tendency caused by the threatening information. Thus, there was no clear conclusion about Hypothesis 4 at this point.

Changes over trials. Again, there was a significant information $\times$ trial interaction $(F(6,108)=2.63, p<.05)$. Just like Experiment $I$, the subjects in the No Inf condition did not show any significant changes in their social comparison responses in process of trials, those in the Enhanc Inf and the Devaluat Inf conditions increased their social comparison responses $(F(3,108)$ $=18.30, p<.005$ for the former; and
$F(3,108)=2.50, p<.07$ for the latter $)$.

\section{Experiment III}

The results of Experiment II give only partial support for our hypotheses about normative social comparison. They predicted that the self-enhancing objective information facilitates social comparison behavior even when the need for self-evaluations has been presumably satisfied, and that the self-devaluating information represses social comparison behavior in the same situation. In normative social comparison, many authors proposed that subjects' self-esteem played a vital role (e.g. Latané, 1966). We propose that people having previously enhanced their selfesteem would facilitate their self-enhancement social comparison when they received the self-enhancing objective information. On the contrary, people who have previously lowered their self-esteem would facilitate their defensive social comparison when they received the self-devaluating objective information; or if possible, they would stop comparing them with others.

We hypothesize that the normative social comparison which was examined in Experiment II will be made saliant by manipulating subjects' self-esteem in advance. Specifically we test the following two hypotheses in this experiment: Social comparison responses following the selfenhancing information will be most predominant for the subjects whose self-esteem has been previously enhanced (Hypothesis 5). On the contrary, defensive suppression of social comparison responses following the self-devaluating information will be most predominant for the subjects whose self-esteem has previously lowered ( $\mathrm{Hy}$ pothesis 6).

\section{Method}

Subjects. The subjects were 73 male and female undergraduates from the same population as the foregoing experiments. Two male subjects who misunderstood the 
instructions were discarded from the analysis of final results.

Design and overview. The level of subjects' self-esteem and the objective information were manipulated in a $2 \times 3$ factorial design. During the first task, the half of the subjects were assigned to high selfesteem (Hi SE) conditions, and the remaining subjects were to low self-esteem (Lo SE) condition. Orthogonally to this treatment, the availability and the contents of objective information were manipulated in the same way as Experiment II.

Procedure. Except for the addition of the following self-esteem manipulation, all procedures were identical with those of Experiment II. The self-esteem of subjects was manipulated by information about their own performances on the first task, or the Kwalwasser-Dykema Music Test. Immediately after the completion of the first task, the answer sheets were collected and marked by one experimenter, while the another experimenter explained the second task. Unlike Experiment II, then, the subjects in this experiment were informed about their scores on the first task. They received their own actual scores. But the norm by which each subject's score was interpreted was misreported to subjects by the following manner. According to the norms of this test for nonmusical Japanese undergraduate, a score of 16 out of 20 is equal to 50th percentile (Tomita \& Kurosu, 1976). Hence, the subjects who scored more than or equal to 16 were assigned to the $\mathrm{Hi} \mathrm{SE}$ condition where all of their scores were interpreted as over 90th percentile. The subjects who got less than 16 were assigned to the Lo SE condition where all of their scores were interpreted as under 20 th percentile. This feedback to each subject was done by circling a corresponding percentile score in one of ten plausible norm distributions which were attached to the answer sheets. All subjects were told that the selection of a particular norm distribution was due to their answers to the face sheet questionnaire of the answer sheets which they had filled out before the first task to assess the diversity of the musical background.

\section{Results and Discussion}

Manipulation checks. The effectiveness of the objective information manipulation was assessed by two items in the post-questionnaire. The subjects' estimates of the percentage of their correct answers in the second task were as follows: The subjects in the Hi SE condition and the Lo $\mathrm{SE}$ condition rated $56.0 \%$ and $42.9 \%$ in the No Inf conditions, $67.7 \%$ and $70.0 \%$ in the Enhanc Inf conditions, and 26.0\% and $23.3 \%$ in the Devaluat Inf conditions. As expected, an analysis of variance indicated a significant main effect of information $(F(2,67)=94,16, p<.005)$. Moreover, the subjects' evaluations about their performances of the second task differed significantly among three information conditions $(F(2,67)=18.69, \quad p<.005) . \quad$ The means were as follows; No Inf conditions, 3.66 for the Hi SE condition, and 3.06 for the Lo SE condition; Enhanc Inf conditions, 3.93 and 4.33; and Devaluat Inf conditions, 2.00 and 2.40. In the light of these results, the manipulation of information was effective although ratings in the Enhanc Inf conditions were not necessarily very high.

Other two items in the post-questionnaire strongly showed that the self-esteem manipulation was successful. All subjects were asked to evaluate their performances of the first task, and to compare them with their subjective confidence on musical ability. In both items, the subjects in the Hi SE condition evaluated their performances as significantly better than those in the Lo $S E$ condition did $(F(2,67)=193.80$, for the former item; and $F(2,67)=55.66$, for the latter. Both $p s<.005)$. Furthermore, there were no significant differences among the subjects in three information conditions in items of their actual performances of the Kwalwasser-Dykema test. 
Table 3

Mean amount of social comparison in Experiment III

\begin{tabular}{lccc}
\hline \multirow{1}{*}{ Groups } & $\mathcal{N}$ & \multicolumn{2}{c}{ Measure } \\
\cline { 3 - 4 } & & Behavioral & Cognitive \\
\hline Hi SE & & \\
$\quad$ No Inf & 11 & 13.81 & 5.18 \\
Enhanc Inf & 15 & 13.53 & 5.13 \\
Devaluat Inf & 10 & 11.20 & 4.90 \\
\hline Lo SE & & & \\
No Inf & 15 & 16.53 & 5.40 \\
Enhanc Inf & 12 & 13.41 & 5.33 \\
Devaluat Inf & 10 & 8.30 & 3.40
\end{tabular}

Social comparison responses. Table 3 shows the mean frequencies of pushing the information request key and the mean ratings of desire to see others' answers. Analyses of variance revealed the significant main effect of information in both indices ( $F$ $(2,67)=3.89$ for the behavioral measure; and $F(2,67)=4.75$ for the cognitive measure. Both $p s<.05$ ). These effects were mainly due to the Lo SE conditions where the subjects in the No Inf condition showed the greatest social comparison responses, followed by the subjects in the Enhanc Inf condition. Social comparison responses in these conditions were significantly greater than those of in the Devaluat Inf condition $(p s<.05$ for both measures by NewmanKeuls tests). These results of the Lo SE conditions were quite similar to those of Experiment II and thereby supported Hypothesis 3. As for the Hi SE conditions, however, this tendency was not significant for both measures. The results of the $\mathrm{Hi}$ SE conditions suggested that the subjects whose self-esteem had been previously enhanced showed some amount of social comparison responses regardless of the information manipulation.

Among the four conditions where the objective information were available, the subjects in the Hi SE-Enhanc Inf condition pushed the information request key mostly, while the least was the subjects in the Lo SE-Devaluat Inf condition. These results tend to support Hypothesis 5 and 6 . However, comparing these data with those of the first two experiments indicated that neither the Hi SE-Enhanc Inf condition nor the Lo SE-Devaluat Inf condition significantly differed from the comparable conditions in Experiment $I$ and II. Thus, it is difficult to conclude that Hypothesis 5 and 6 were confirmed. The present data may suggest that any clues which enhance subjects' self-concept will facilitate social comparison responses. In other words, either the self-enhancing objective information or the previous experience which enhanced subjects' self-esteem is a sufficient determinant to trigger social comparison responses. It might be due to a ceiling effect that the effects of these two determinants are not in a cumulative manner. The present data also suggested that there was no clear evidence of the suppression of social comparison responses in these experiments.

Changes over trials. Changes in pushing the information request key over trials were replicated in this experiment as in the previous two experiments reported in this paper. The information $\times$ trial interaction was significant $(F(6,201)=2.14, p<$ $.05)$. The subjects for whom the objective information was available showed a significant increase in social comparison responses with time (as for the Enhanc Inf condition, $F(3,201)=4.82$ for the Hi SE and $F(3,201)=5.40$ for the Lo SE; and as for the Devaluat Inf conditions, $F(3,201)$ $=9.98$ for the $\mathrm{Hi} \mathrm{SE}$ and $F(3,201)=6.11$ for the Lo SE. All $p s<.01)$. On the contrary, no significant changes over trials were found in the No Inf conditions. This consistent result strongly suggests that there are different kinds of social comparison in terms of the availability of the objective information.

General Discussion

The results obtained in the present three 
experiments reveal the following three points: First, social comparison responses generally occurs when the objective information providing the criterion for judging performance is unavailable, especially in the case that such criterion itself depends on social consensus. Second, even if the objective information is available, a considerable amount of social comparison occurs, especially when the subjects' selfesteem is enhanced by the objective information or when it has been increased in advance. Third, social comparison responses increase over time when objective information is available, whereas no such increase is found when the objective information is unavailable.

Social comparison behavior due to the unavailability of the objective information seems to function as an informational basis for self-evaluation by which people assess the correctness of their answers and further assess their own musical ability. This interpretation is confirmed by an additional analysis of correlational data. As mentioned earlier, subjects indicated their confidence in their answers on each trial. The mean of those 20 confidence ratings was positively correlated with the frequency of pushing the information request key in the conditions which almost completely lacked in any given objective means for self-evaluation, namely in the No Inf conditions of Experiment I and II $(r=.77-$ $.24)$; although the only significant correlation was that in the Physic condition of Experiment $I(t=4.18, p<.002)^{\text {? }}$. Thus, in

7 By contrast, no or slightly negative correlations (.04- - .34) were found in the conditions where the objective, especially enhancing, information was available (the Inf conditions in Experiment I, the Enhanc Inf conditions of Experiments II and III), or where some enhancing clues were given (the $\mathrm{Hi}$ SE-No Inf condition in Experiment III). But when the self-devaluating information or some threatening clues were given (the Devaluat Inf conditions in Experiments II and III, and the Lo SE-No Inf condition in Experiment III), the correlations were positive $(.65-.29)$ as discussed later. these conditions, it is possible to say that the more subjects compare their answers with others', the more they increase their confidence in their answers. This interpretation also support the Festinger's $\mathrm{Hy}_{\text {- }}$ pothesis II.

The considerable amount of social comparison behavior found in the Enhanc Inf conditions in Experiment II and III cannot be interpreted by Festinger's selfevaluation notion. It is because the subjects in these conditions still showed a considerable amount of social comparison even when their immediate self-evaluation need has been presumably satisfied with the objective information. This suggests that the purpose of these social comparison is not uncertainty reduction but the enhancement of one's self-esteem, and that the enhancing self-esteem is accomplished by means of validating their excellent performances through contrasting with those of other participants. In fact, there were no positive correlations between subjects' confidence in their answers and the frequency of social comparison responses in the Enhanc Inf conditions (see footnote 7). Thus, we conclude that the social comparison in these conditions represents the normative function of social comparison by which people keep their self-esteem in a positive fashion to conform to the expectation of themselves and other person.

The lack of clear evidence for the defensive suppression of social comparison in the Devaluat Inf conditions in Experiment II and III disconfirmed our Hypothesis 4 and 6. Brickman \& Bulman (1977) pointed out that the desire to avoid social comparison was typically facilitated more in public situations than in private ones. In the present experiments, the subjects seating in individual cubicles remained almost completely anonymous so that their desire to avoid social comparison might be minimum. Moreover, the social comparison behavior observed in the Devaluat Inf conditions may be interpreted as that the subjects who received succesive negative infor- 
mation pushed the information request key to newly aroused uncertainty due to the objective information itself. This interpretation may be partially supported by the correlational data which indicated the similarity of the relationship between confidence and social comparison in the Devaluat Inf conditions to that in the No Inf conditions. In the case that subjects received self-devaluating information in Experiment II and III, the correlations between confidence and social comparison were positive (a significant correlation was found in Experiment II $(t=2.26, p<.05))$. In line with this interpretation, Brickman and Berman (1971) reported generally prolonged tendency of information search by the subjects who received low scores in a private examination situation.

In the light of the discussions thus far, the prevailing information $\times$ trial interaction found in all three experiments may be interpreted as follows: During the first few trials the subjects who received the objective information appear to estimates their performances through the objective information. Then, the subjects in the Enhanc Inf conditions gradually begin to compare their answers with others' in order to acquire the information which enables them to enhance their self-esteem further. The subjects in the Devaluat Inf conditions might do so in order to reduce the newly developed uncertainty or to find similar others. In either condition, social comparison behavior would be increased over time. By contrast, the subjects in the No Inf conditions seem to be concerned with comparing their answers with others' from the very early trials to evaluate the correctness of their own answers because of the unavailability of the objective information. Thus, it should be noted that there were some conditions under which people showed social comparison behavior in spite of the availability of the objective mean for self-evaluation. We think this is a indication of the second type of social comparison which does not serve for self-eval- uation.

These interpretations suggest the importance of studying the agreement between the subjects' own answers and others'. In the Enhanc Inf conditions, the subjects might be pleased if their answers disagree with others' answers. Both in the No Inf and the Devaluat Inf conditions, the subjects might be rewarded by the agreement of their answers with others' answers. The agreement between the subject's answer and what appeared to be others' was not experimentally manipulated but randomized in the present experiments. Post-hoc analysis revealed that there was significant positive correlation between the frequency of pushing the information request key and the degree of the agreement between the subject's answers and others' in certain conditions (e.g. the Soc-No Inf condition of Experiment I; Takata, Hayashi, \& Matsumoto, 1977). This result seems to support so called " similality hypothesis" in Festinger's original theory. On this point, however, further study is needed to clarify the effect of the agreement and similarity upon the social comparison behavior in the present experimental paradigm.

\section{References}

Brickman, P., \& Berman, J.J. 1971 Effects of performance expectancy and outcome certainty on interest in social comparison. Journal of Experimental Social Psychology, 7, 600-609.

Brickman, P., \& Bulman, R.J. 1977 Pleasure and pain in social comparison. In J. M. Suls \& R. L. Miller (Eds.), Social comparison processes: Theoretical and empirical perspectives. Washington, D. C.: Hemisphere/ Halsted. Pp. 149-186.

Deutsch, M., \& Gerard, H. B. 1955 A study of normative and informational social influences upon individual judgement. Journal of Abnormal and Social Psychology, 51, 629-636.

Festinger, L. 1954 A theory of social comparison processes. Human Relations, 7, 117-140.

Gerard, H. B., \& Rabbie, J. M. 1961 Fear and social comparison. Journal of Abnormal and Social Psychology, 62, 586-592.

Goethals, G. R., \& Darley, J.M. 1977 Social 
comparison theory: An attributional approach. In J. M. Suls \& R. L. Miller (Eds.), Social comparison processes: Theoretical and empirical perspectives. Washington, D. C.: Hemisphere/ Halsted. Pp. 259-278.

Latané, B. 1966 Studies in social comparison: Introduction and overview. Journal of Experimental Social Psychology, Supplement 1, 1-5.

Kwalwasser, J., \& Dykema, P. W. 1930 K-D music test: Manual of direction. New York: Carl Fisher.

Radloff, R. 1961 Opinion evaluation and affiliation. Joumal of Abnormal and Social Psychology, 62, 578-585.

Seashore, C. E., Lewis, D., \& Saetveit, J. C. 1960 Seashore Measures of Musical Talents: 1960 Revised Manual. New York: The Psychological Corporation.

Singer, J.E., \& Shockley, V. L. 1965 Ability and affiliation. Journal of Personality and Social Psychology, 1, 95-100.

Suls, J. M., \& Miller, R. L. (Eds.) 1977 Social comparison processes: Theoretical and empirical perspectives. Washington, D. C.: Hemisphere/ Halsted.
Takata, C., \& Takata, T. 1976 The influence of models on the evaluation of ability: Two functions of social comparison processes. Japanese Journal of Psychology, 47, 74-84. (In Japanese with English summary)

Takata, T. 1971 Effects of social comparison processes as determinants of affiliation. Waseda Psychological Report, 3, 10-16. (In Japanese)

Takata, T. 1974 A fundamental study on the theory of social comparison processes: I. Japanese Journal of Experintental Social Psychology, 14, 132-138. (In Japanese with English summary) Takata, T., Hayashi, H., \& Matsumoto, Y. 1977 A fundamental study on the theory of social comparison processes: V. Proceedings of the 25th Annual Convention of the Japanese Group Dynamics Association, 2-3. (In Japanese)

Tomita, M., \& Kurosu, M. 1976 A preliminary study concerning the assessment of musical aptitude. Waseda Psychological Report, 8, 15-29. (In Japanese)

Wing, H. 1960 Manual for Wing standardised tests of musical intelligence. Windsor, England: NFER Publishing.

(Received Jan. 8, 1981; accepted March 28, 1981) 